\title{
Co-Design of Controller and Communication Topology for Vehicular Platooning
}

\author{
Amir Firooznia, Student Member, IEEE, Jeroen Ploeg, Nathan van de Wouw, Member, IEEE, \\ and Hans Zwart, Member, IEEE
}

\begin{abstract}
Small inter-vehicle distances can increase traffic throughput on highways. Human drivers are not able to drive safely under such conditions. To this aim, cooperative adaptive cruise control (CACC) systems have been developed, which require vehicles to communicate with each other through a wireless communication network. By communicating control-relevant information, the vehicles equipped with the CACC system are able to react more quickly to disturbances caused by preceding vehicles and, therefore, are able to maintain the desired (small) inter-vehicle distance while avoiding string instability. String stability relates to the propagation of the effect of disturbance on system states over the vehicle string. Commonly used approaches to design controllers yielding string stability, involve an iterative process requiring an a priori designed controller with a priori defined communication topology. The main contribution of this paper is to propose a synthesis strategy for both local controllers and the communication structure, while guaranteeing string stability for infinite-length vehicular strings. The obtained results are illustrated by model-based case studies.
\end{abstract}

Index Terms - Vehicular strings, string stability, optimal control.

\section{INTRODUCTION}

$\mathbf{R}$ ECENTLY, the capacity of highways has turned out to be a limiting factor for traffic throughput, which regularly causes traffic jams. The road capacity can be increased by reducing the inter-vehicle distance while maintaining the same velocity level. Since this would be unsafe in case of human drivers, vehicle automation in longitudinal direction is required to ensure traffic safety while pursuing a reduction of the intervehicle distance. Adaptive Cruise Control (ACC) is an example of such longitudinal vehicle automation [7], [8]. Currently, ACC functionality is widespread and available in numerous

Manuscript received June 30, 2016; revised January 11, 2017; accepted January 14, 2017. The Associate Editor for this paper was P. Ioannou.

A. Firooznia is with the Delft Center for Systems and Control, Delft University of Technology, 2628 CD Delft, The Netherlands (e-mail: a.firooznia@tudelft.nl).

J. Ploeg is with the Integrated Vehicle Safety Department, TNO, 5700 AT Helmond, The Netherlands (e-mail: jeroen.ploeg@tno.nl).

N. van de Wouw is with the Mechanical Engineering Department, Eindhoven University of Technology, 5600 MB Eindhoven, The Netherlands; with the Delft Center for Systems and Control, Delft University of Technology, 2628 CD Delft, The Netherlands; and also with the Department of Civil, Environmental and Geo-Engineering, University of Minnesota, Minneapolis, MN 55455 USA (e-mail: n.v.d.wouw@tue.nl).

H. Zwart is with the Faculty of Electrical Engineering, Mathematics and Computer Science, Department of Applied Mathematics, University of Twente, 7522 NB Enschede, The Netherlands, and also with the Mechanical Engineering Department, Eindhoven, Eindhoven University of Technology, 5600 MB Eindhoven, The Netherlands (e-mail: h.j.zwart@utwente.nl).

Digital Object Identifier 10.1109/TITS.2017.2660544 commercial vehicles. ACC-equipped vehicles automatically keep a desired distance to the preceding vehicle, or in the absence of one, realize a desired velocity [24]. For the purpose of ACC, the inter-vehicle distance and the relative velocity to the preceding vehicle is measured by a radar or a scanning laser (lidar). The current ACC systems primarily focus on driving comfort and, to a smaller degree, on traffic safety. Therefore, relatively large inter-vehicle distances are adopted in commercially available systems, thereby limiting traffic throughput for ACC-based systems [22].

A more advanced version of ACC is called Cooperative Adaptive Cruise Control (CACC). Vehicles, that are equipped with CACC, not only measure the relative distance and velocity with respect to the preceding vehicle, but can also communicate to other CACC instrumented vehicles by means of a wireless network. The additional information obtained through wireless communication is a key enabler for the design of other controllers that allow for smaller inter-vehicular distances, while still guaranteeing string stability (in comparison to ACC) [15], [16], [23]. An additional advantage of CACC is that the fuel consumption will be reduced because variations in velocity are decreased. Moreover, small intervehicle distances reduce the aerodynamic drag, in particular for trucks and therewith can reduce a significant reduction in fuel consumption, as in [1].

In the automation of vehicular strings, the ability to attenuate the effect of disturbances along the string is a key requirement. Namely, so-called ghost traffic jams are generally the consequence of the amplification of the effects of disturbances (e.g. induced by human drivers) along the vehicle string. The property related to the attenuation of the effects of disturbances along the vehicle string in upstream direction is often referred to as string stability (see [9], [11]-[13]). Contrary to conventional stability definitions for dynamical systems, which are concerned with the evolution of system responses over time, string stability for vehicle strings focuses on the propagation of system states over the vehicle index. Different definitions of string stability are given in the literature (e.g. see [17], [18], [20]), focusing on a specific type of perturbations or on specific interconnection topologies. In [20], for instance, a Lyapunov stability approach is used to analyze string stability, which only focuses on initial condition perturbations. As another example, string stability definitions in the frequency domain are employed to analyze string stability in the presence of external perturbations, see e.g. [16]-[18]. 
Three commonly used definitions for string stability are the time-domain definition [21], the frequency-domain definition [13], and the z-domain definition [2], [3]. The timedomain approach focuses on initial condition perturbations, while the frequency-domain approach focuses on the amplification of (external) disturbances. These two definitions have been used for analyzing string stability of vehicular strings with relatively simple and a priori fixed communication topologies (e.g. one vehicle look-ahead [13]). However, applying time-domain or frequency-domain definitions of string stability to spatially invariant system models of infinite vehicle strings makes the stability analysis rather intricate for complicated vehicle interconnections (communication topologies), such as multiple vehicles look-ahead topologies, which allow for both look behind and ahead. Hence, these two approaches can be used for analyzing string stability of the vehicular strings with relatively simple and predefined communication topologies (e.g. one vehicle look-ahead [13]). In these methods, a controller is typically designed for a predefined communication topology, where the communication topology is determined in a heuristic way. Here, the communication topology refers to the structure of communication in between vehicles in the CACC setup. In other words, the communication topology is chosen a priori, which results in exclusion of a larger solution space for the optimal performance. Subsequently, string stability of the closed-loop system can be analyzed a posteriori and if it is not string stable, then another iteration of controller design is required. This may be a time consuming and non-optimal approach towards the design of controllers guaranteeing string stability. Therefore, a co-design approach for vehicle-following controllers and their underlying communication topology is required, such that it guarantees string stability of the resulting networked closed-loop vehicular platooning system. As will be shown in this paper, the $z$-domain approach for spatially invariant system models for (infinite) vehicle strings allows co-design of controller and communication topology, while none of the existing methods supports such co-design.

In this paper, we present an approach for the co-design of the vehicle following controller and the communication topology jointly guaranteeing string stability, thereby also providing insight into the effects of the communication topology on the controller performance. In the proposed approach, an optimal control problem setting is considered for an infinite length vehicle string, in which the vehicle string is considered as an spatially invariant system in one dimension. Previously, in [4], the analysis and synthesis of distributed controllers for spatially interconnected systems are considered for a class of spatially invariant systems with an $\ell_{2}$-induced norm as the performance criterion. In [10], an optimal localized controller is designed for a vehicular string considered as a spatially invariant system with communication allowed only with the immediate neighbors. Moreover, in [5], a state-space description of systems in which information propagates at most one unit in space for every unit in time was provided and relaxations were used to obtain suboptimal controllers. However, in the existing literature of controller synthesis for spatially invariant systems, the communication topologies are

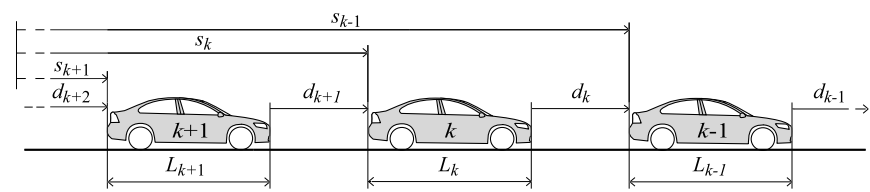

Fig. 1. A string of vehicles.

fixed a priori, hence not exploiting the possibility of co-design of the controller and the communication topology.

The contribution of the current paper is the development of a synthesis strategy for both distributed controllers and the communication topology that guarantees string stability of the automated vehicular string. To this end, an optimal control strategy will be employed that is applicable to the model of the vehicle string dynamics in the so-called, 'spatialfrequency domain'. The methodology is tested via simulation case studies.

The paper is organized as follows. We consider the vehicle modeling and problem formulation in Section II. The vehicle string model and the concept of stability in the spatialfrequency domain (used to assess string stability) are then presented in Section III. The co-design of the controllers and communication topology is treated in Section IV. In Section V, the simulation results are presented for a representative case study. We conclude the paper in Section VI with a brief summary of our contributions followed by a discussion.

\section{Vehicle Modeling And Problem Formulation}

\section{A. Vehicle Model}

Consider a string of vehicles as shown in Fig. 1, where each vehicle is assigned with an index $k$ increasing in upstream direction. Here, $L_{k}$ represents the length of vehicle $k$ and the absolute position of the rear bumper of vehicle $k$ is denoted by $s_{k}$. The distance $d_{k}$ between the vehicle $k$ and the preceding vehicle $k-1$ equals

$$
d_{k}=s_{k-1}-s_{k}-L_{k}
$$

The distance error $e_{k}$ is defined as:

$$
e_{k}=d_{k}-d_{r, k},
$$

where $d_{r, k}$ is the desired headway of vehicle $k$ which will be defined via a so-called 'spacing policy'. Assuming tight vehicle following, where the speed difference between the vehicles is relatively small compared to the absolute speed, the desired headway can be formulated as follows [14]:

$$
d_{r, k}=r_{k}+h_{k} v_{k},
$$

where $r_{k}$ is called the standstill distance and $h_{k}$ is known as the time gap. A constant time headway spacing policy corresponds to the case $h_{k}=h$ for all $k$ with $h$ constant.

The dynamic behavior of the vehicles can be modeled with a single vehicle model by using appropriate low-level vehicle acceleration controllers that compensate differences in the vehicles' dynamics in the string. From now on, we therefore consider the vehicle string to be homogeneous. We employ a 
simplified vehicle model represented by [19]:

$$
\left(\begin{array}{c}
\dot{s}_{k} \\
\dot{v}_{k} \\
\dot{a}_{k}
\end{array}\right)=\left(\begin{array}{c}
v_{k} \\
a_{k} \\
-\tau^{-1} a_{k}+\tau^{-1} u_{k}
\end{array}\right) \text {, }
$$

where $v_{k}$ is the vehicle speed, $a_{k}$ is its acceleration, $u_{k}$ is the external input being the controlled acceleration, and $\tau$ is a time constant representing the engine dynamics. However, as the inter-vehicle distances are more relevant than the absolute vehicle positions, the vehicle model (4) can be rewritten with distance $d_{k}$ as a state, instead of the position $s_{k}$, as follows:

$$
\left(\begin{array}{c}
\dot{d}_{k} \\
\dot{v}_{k} \\
\dot{a}_{k}
\end{array}\right)=\left(\begin{array}{c}
v_{k-1}-v_{k} \\
a_{k} \\
-\tau^{-1} a_{k}+\tau^{-1} u_{k}
\end{array}\right)
$$

where (1) and (4) were used. From now on, we denote the state by $x_{k}:=\left[\begin{array}{lll}d_{k} & v_{k} & a_{k}\end{array}\right]^{T}$. The equilibrium state of (5) for $u_{k}=0$ satisfies

$$
x_{k_{e q}}=\left(\begin{array}{l}
d_{k_{e q}} \\
v_{k_{e q}} \\
a_{k_{e q}}
\end{array}\right)=\left(\begin{array}{c}
C_{k} \\
v_{k-1} 1_{e q} \\
0
\end{array}\right)=\left(\begin{array}{c}
C_{k} \\
v_{e q} \\
0
\end{array}\right),
$$

where $C_{k}$ is a constant that can be different for each vehicle. So, (6) expresses that, in the equilibrium state, all vehicles are moving with equal constant velocity $v_{e q}$ and a constant distance $C_{k}$ to the preceding vehicle. As a next step, we pursue a state transformation to perturbation coordinates $\left(e_{k} v_{k}^{\prime} a_{k}\right)$, with respect to the equilibrium state in (6), where $e_{k}=d_{k}$ $r_{k}-h v_{k}$, see (2) and (3) with $h$ constant, and $v_{k}^{\prime}=v_{k}-v_{e q}$. This leads to the following state-space model:

$$
\left(\begin{array}{c}
\dot{e}_{k} \\
\dot{v}_{k}^{\prime} \\
\dot{a}_{k}
\end{array}\right)=\left(\begin{array}{c}
v_{k-1}-v_{k}-h a_{k} \\
a_{k} \\
-\frac{1}{\tau} a_{k}+\frac{1}{\tau} u_{k}
\end{array}\right)=\left(\begin{array}{c}
v_{k-1}^{\prime}-v_{k}^{\prime}-h a_{k} \\
a_{k} \\
-\frac{1}{\tau} a_{k}+\frac{1}{\tau} u_{k}
\end{array}\right) .
$$

The benefit of this state transformation is, firstly, that the constant time headway policy is now embedded in the dynamics and, secondly, that the vehicle following error $e_{k}$ is an explicit state.

\section{B. Problem Formulation}

Considering a string of vehicles, the goal of automatic vehicle control is to increase the traffic throughput, which can be done by decreasing the inter-vehicle distances $d_{k}$. However, small distances could cause a so-called 'shockwave' leading to a decrease in traffic throughput [13]. In order to achieve vehicle following behavior $\left(e_{k}(t) \rightarrow 0\right.$ as $t \rightarrow \infty$ with $e_{k}$ as in (2)) while avoiding such 'shockwaves', the control problem consists of two objectives: vehicle following and string stability.

The vehicle following objective, which is often related to individual vehicle stability [6], aims to ensure that each vehicle follows the preceding vehicle at a desired distance in the absence of perturbations, which relates to the stability property of (6) for the dynamics (5) in closed loop with a controller for $u_{k}$ (to be designed). String stability is related to how the responses of the vehicles to perturbations evolve not only in time, but also in the vehicle index (i.e., along the string of vehicles). More precisely, the ability of the string of vehicles to attenuate the effect of disturbances caused by an arbitrary vehicle in the string as we move away (in terms of the vehicle index) from that vehicle, is called string stability. In this paper, the definition for string stability in the $z$-domain (see [3]) will be employed. Here, the challenge to be tackled is to design a stabilizing controller for the vehicular string in the $z$-domain with guaranteeing string stability and at the same time determining a suitable communication topology for the string. Thereafter, the obtained controllers and communication topologies in the $z$-domain have to be transformed into the spatial domain.

Loosely speaking the $z$-domain is the discrete 'frequency domain' related to the vehicle index of the (infinite) sequence of vehicles. In the next section, we introduce the vehicle string model and the notion of string stability (with respect to initial condition perturbations) in the $z$-domain. Next, based on the resulting $z$-domain vehicular string model, we present a co-design approach for the vehicle controllers and the communication topology in Section IV. The $z$-domain approach involves the modeling of the vehicular string string as being infinitely long. In practice, the length of the vehicular string is clearly bounded. Therefore, in Section IV and V, we show how the insights obtained through the controller synthesis for infinite strings can also be used to design controllers inducing string stability for finite vehicle strings.

\section{Vehicular String Modeling and StRing STABILITY IN THE $z$-DOMAIN}

\section{A. Vehicular String Modeling in the z-Domain}

Let us now model the (infinite) vehicle string dynamics in terms of an infinite-dimensional model with spatially invariant dynamics. Hereto, we employ the following class of infinitedimensional systems with spatially invariant dynamics [2] written in state-space form as follows:

$$
\begin{aligned}
\frac{d}{d t} x_{k}(t) & =\sum_{j=-\infty}^{\infty} A_{k-j} x_{j}(t)+B_{k-j} u_{j}(t) \\
y_{k}(t) & =\sum_{j=-\infty}^{\infty} C_{k-j} x_{j}(t)
\end{aligned}
$$

where the vectors $x_{k}(t) \in \mathbb{R}^{n}, u_{k}(t) \in \mathbb{R}^{m}$, and $y_{k}(t) \in \mathbb{R}^{p}$ denote the state, the control input, and the output vectors of the $k$-th subsystem, with $k \in \mathbb{Z}$, at time $t \geq 0$, respectively. Moreover, the matrices $A_{k-j} \in \mathbb{R}^{n \times n}, B_{k-j} \in \mathbb{R}^{n \times m}$, and $C_{k-j} \in \mathbb{R}^{p \times n}$ describe the influence of subsystem $j$ on subsystem $k$. System (8) is called spatially invariant since the influence between subsystems depends only on the difference between their indices, or, in other words, a shift in the numbering of the subsystems does not change the overall dynamics.

Note that vehicular strings with homogeneous vehicle dynamics, i.e., constant $\tau$ in (7), and constant time gap policy, i.e., constant $h_{k}=h$ in (3), can be modeled as spatially invariant systems represented in (8).

It is essential to note that the state space, the input space, and the output space of system (8) are taken to be square summable i.e. $x(t):=\left(x_{k}(t)\right)_{k=-\infty}^{\infty} \in \ell_{2}\left(\mathbb{C}^{n}\right)$, 
$u(t):=\left(u_{k}(t)\right)_{k=-\infty}^{\infty} \in \ell_{2}\left(\mathbb{C}^{m}\right)$, and $y(t):=\left(y_{k}(t)\right)_{k=-\infty}^{\infty} \in$ $\ell_{2}\left(\mathbb{C}^{p}\right)$, where $\ell_{2}\left(\mathbb{C}^{\cdot}\right)$ are the square summable vectors, see e.g. [3] and $\left(\psi_{k}(t)\right)_{k=-\infty}^{\infty}:=\left(\ldots, \psi_{-1}^{T}(t), \psi_{0}^{T}(t), \psi_{1}^{T}(t), \ldots\right)^{T}$ for any $\psi_{k}(t) \in \mathbb{C}^{n}$ (see Appendix VI). We stress that the squaresummability assumption will eliminate some states from the state-space of (8).

For the purpose of the analysis of string stability (see the end of this section for the formal definition of string stability) and controller synthesis for string stability (see Section IV), we subject system (8) to the so-called bilateral $\mathcal{Z}$-transformation, which facilitates the co-design of a string stable controller and the corresponding communication topology. The bilateral $\mathcal{Z}$-transformation transforms the vector $x(t)=\left(x_{k}(t)\right)_{k=-\infty}^{\infty}$ to the function $\breve{x}(z, t)$, defined as follows:

$$
\check{x}(z, t)=\mathcal{Z}[x(t)](z)=\sum_{k=-\infty}^{\infty} x_{k}(t) z^{-k} .
$$

Due to the property of the bilateral $\mathcal{Z}$-transformation, $z$ may be interpreted as the shift operator. Here, the $z$ variable is not used as a time shift operator, but as a system (i.e., vehicle) index shift operator. Now, knowing that $\mathcal{Z}[x(t)](z)=\check{x}(z, t)$ where $z=e^{j \theta}$ for $\theta \in[0,2 \pi]$, the state-space system (8), can be rewritten in the $z$-domain by using:

$$
\begin{aligned}
\mathcal{Z} & {\left[\frac{d}{d t} x(t)\right](z)=\mathcal{Z}\left[\frac{d}{d t}\left(x_{k}(t)\right)_{k=-\infty}^{\infty}\right](z) } \\
= & \mathcal{Z}\left[\left(\sum_{j=-\infty}^{\infty} A_{k-j} x_{j}(t)+B_{k-j} u_{j}(t)\right)_{k=-\infty}^{\infty}\right](z) \\
= & \sum_{k=-\infty}^{\infty} \sum_{j=-\infty}^{\infty}\left(A_{k-j} x_{j}(t)+B_{k-j} u_{j}(t)\right) z^{-k} \\
= & \sum_{k=-\infty}^{\infty} \sum_{j=-\infty}^{\infty}\left(A_{k-j} z^{-(k-j)} x_{j}(t)+B_{k-j} z^{-(k-j)} u_{j}(t)\right) z^{-j} \\
= & \sum_{j=-\infty}^{\infty} \sum_{i=-\infty}^{\infty}\left(A_{i} z^{-i} x_{j}(t)+B_{i} z^{-i} u_{j}(t)\right) z^{-j} \\
= & \sum_{i=-\infty}^{\infty} A_{i} z^{-i} \sum_{j=-\infty}^{\infty} x_{j}(t) z^{-j}+\sum_{i=-\infty}^{\infty} B_{i} z^{-i} \sum_{j=-\infty}^{\infty} u_{j}(t) z^{-j} \\
= & \check{A}(z) \check{x}(z, t)+\check{B}(z) \check{u}(z, t),
\end{aligned}
$$

where $\breve{A}(z):=\sum_{i=-\infty}^{\infty} A_{i} z^{-i}$ and $\check{B}(z):=\sum_{i=-\infty}^{\infty} B_{i} z^{-i}$. Similar as in (10), the state-space system (8) with output, can be transformed into the $z$-domain, leading to the following system dynamics in the $z$-domain:

$$
\begin{aligned}
\frac{d}{d t} \check{x}(z, t) & =\check{A}(z) \check{x}(z, t)+\check{B}(z) \check{u}(z, t) \\
\check{y}(z, t) & =\check{C}(z) \check{x}(z, t),
\end{aligned}
$$

where $\check{A}, \check{B}$, and $\check{C}$ are multiplicative operators, since their action is pointwise multiplication. Now, $\ell_{2}\left(\mathbb{C}^{n}\right)$ is isometrically isomorphic ${ }^{1}$ to $\mathcal{L}_{2}\left(\partial \mathbb{D} ; \mathbb{C}^{n}\right)^{2}$ under the bilateral

\footnotetext{
${ }^{1}$ Two isometrically isomorphic normed vector spaces share the same structure, so they are usually identified with each other.

$2 \check{\psi} \in \mathcal{L}_{2}\left(\partial \mathbb{D} ; \mathbb{C}^{r}\right)$ is a mapping from $\partial \mathbb{D}$ to $\mathbb{C}^{r}$ for which $\|\check{\psi}\|_{\mathcal{L}_{2}}<\infty$ (see Appendix VI).
}

$\mathcal{Z}$-transformation, i.e., $\|x\|_{\ell_{2}\left(\mathbb{C}^{n}\right)}=\|\check{x}\|_{\mathcal{L}_{2}\left(\partial \mathbb{D} ; \mathbb{C}^{n}\right)}$, where $\partial \mathbb{D}:=\{z \in \mathbb{C}|| z \mid=1\}$ is the unit circle. Hence, the state linear system $\sum_{i}\left(A_{i}, B_{i}, C_{i}, 0\right)$ is isometrically isomorphic to the state linear system $\sum(\check{A}, \check{B}, \check{C}, 0)$ on the state-space $\mathcal{L}_{2}\left(\partial \mathbb{D} ; \mathbb{C}^{n}\right)$ with the input and output spaces $\mathcal{L}_{2}\left(\partial \mathbb{D} ; \mathbb{C}^{m}\right)$ and $\mathcal{L}_{2}\left(\partial \mathbb{D} ; \mathbb{C}^{p}\right)$, respectively.

In this study, we assume that $\check{A}, \check{B}$, and $\check{C}$ are all bounded operators. Here, $\check{O}(z)$ defines a bounded operator from $\mathcal{L}_{2}\left(\partial \mathbb{D} ; \mathbb{C}^{r}\right)$ to $\mathcal{L}_{2}\left(\partial \mathbb{D} ; \mathbb{C}^{s}\right)$ if and only if $\check{O} \in \mathcal{L}_{\infty}\left(\partial \mathbb{D} ; \mathbb{C}^{s \times r}\right)^{3}[3]$.

Note that the equilibrium state of the system (5) with zero input $u_{k}=0$ is given in (6). For non-zero values of the equilibrium velocity $v_{e q} \neq 0$ in $(6), x_{e q}:=\left(x_{i_{e q}}\right)_{i=-\infty}^{\infty} \notin$ $\ell_{2}\left(\mathbb{C}^{n}\right)$, which means that this equilibrium is not in the statespace unless $v_{e q}=0$. Furthermore, a similar statement is true for $C_{k}$ (see (6)). Hence, the modified states in (7) are chosen such that the resulting equilibrium satisfies the squaresummability without loosing the practical feasibility of the control problem and subsequently the $\mathcal{Z}$-transformation is applied to system (7), which yields the following vehicle string model in the $z$-domain:

$$
\begin{aligned}
\frac{d}{d t}\left(\begin{array}{l}
\check{e}(z, t) \\
\check{v}^{\prime}(z, t) \\
\check{a}(z, t)
\end{array}\right) \\
\quad=\left(\begin{array}{ccc}
0 & -1+z^{-1} & -h \\
0 & 0 & 1 \\
0 & 0 & -\frac{1}{\tau}
\end{array}\right)\left(\begin{array}{l}
\check{e}(z, t) \\
\check{v}(z, t) \\
\check{a}(z, t)
\end{array}\right)+\left(\begin{array}{l}
0 \\
0 \\
\frac{1}{\tau}
\end{array}\right) \check{u}(z, t),
\end{aligned}
$$

which is of the form of $\frac{d}{d t} \check{x}(z, t)=\breve{A}(z) \check{x}(z, t)+\check{B} \breve{u}(z, t)$ with a state vector $\check{x}(z, t)=\left[\check{e}(z, t) \check{v}^{\prime}(z, t) \check{a}(z, t)\right]^{T}$.

\section{B. String Stability in the z-Domain}

Now, the state-space system (8) has been transformed into $z$-domain as in (11), and string stability can effectively defined in the z-domain [2], [3] (also see Appendix VI).

Definition 1 (String stabilizability in the z-domain): Consider an infinite-dimensional model with spatially invariant dynamics described by (8), such that after application of the bilateral $\mathcal{Z}$-transformation, it is expressed as system (11). Then, the model (8) is called exponentially/ asymptotically stabilizable when there exists a state feedback $u_{k}(t)=-\sum_{j=-\infty}^{\infty} L_{k-j} x_{j}(t)$ such that the closedloop system is exponentially/asymptotically stable according to Definitions 5 and 6 , where $L_{j}$ and $\breve{L}(z)$ are related via $\mathcal{Z}$-transformation (see Appendix VI), where $\check{L}(z)$ is the control gain in the $z$-domain associated with the following control law in the $z$-domain $\breve{u}(z, t)=-\check{L}(z) \check{x}(z, t)$.

Note that a string-stabilized vehicular string as described in Definition 1 is equivalent to a string that not only attenuates any introduced initial condition perturbation (with respect to the equilibrium) in time, but also over the vehicle index. In other words, deviations from an equilibrium state will not only decrease in time, but the propagation of that disturbance along the vehicle index will vanish in spatial direction.

\footnotetext{
$3 \check{\Phi} \in \mathcal{L}_{\infty}\left(\partial \mathbb{D} ; \mathbb{C}^{r \times s}\right)$ is a mapping from $\partial \mathbb{D}$ to $\mathbb{C}^{r \times s}$ for which $\|\check{\Phi}\|_{\infty}<\infty$ (see Appendix VI).
} 


\section{Optimal CONTROLleR Synthesis}

In this section, we consider the optimal control co-design with guaranteed string stability for a vehicular problem in the framework of spatially invariant systems. Definition 1 of string stabilizability will be used to perform string stability analysis of infinite vehicle strings and it will serve as a basis for the synthesis of a co-design strategy for the vehicle controllers and the underlying communication topology.

\section{A. Optimal Controller Design For the Case of Spatially Invariant Systems}

In order to design an optimal linear quadratic controller, the pair $\{\check{A}(z), \check{B}\}$ should be stabilizable, which can be checked by analyzing the controllability matrix. Using the Popov-Belevitch-Hautus stabilizability condition [3], it can be shown that the pair $\left\{\check{A}_{z=1}, \check{B}\right\}$ is not exponentially stabilizable. However, as we will show below, we can still employ optimal controller synthesis to asymptotically stabilize system (12) and consequently render system (7) asymptotically (string) stable [25].

To design a controller in the $z$-domain, the following cost function is considered:

$$
\begin{aligned}
\check{J}= & \frac{1}{2 \pi} \int_{0}^{2 \pi} \int_{0}^{\infty} \check{x}\left(e^{j \theta}, t\right)^{*} \check{Q}\left(e^{j \theta}\right) \check{x}\left(e^{j \theta}, t\right) \\
& +\check{u}\left(e^{j \theta}, t\right)^{*} \check{R}\left(e^{j \theta}\right) \check{u}\left(e^{j \theta}, t\right) d t d \theta,
\end{aligned}
$$

in which $\check{Q}\left(e^{j \theta}\right) \geq 0, \check{R}\left(e^{j \theta}\right)>0$ for all $\theta$ in $[0,2 \pi]$ and $x^{*}$ denotes the complex conjugate transpose of a vector $x$. Then, in order to minimize $\breve{J}$, the following Algebraic Riccati Equation

$$
\begin{aligned}
& \check{P}(z) \check{A}(z)+\check{A}(z)^{*} \check{P}(z) \\
& \quad-\check{P}(z) \check{B} \check{R}(z)^{-1} \check{B}^{T} \check{P}(z)+\check{Q}(z)=0,
\end{aligned}
$$

has to be solved pointwise in the complex domain $\mathbb{C}$ where $z=e^{j \theta}, \theta \in[0,2 \pi]$, and $\check{P}(z)=\check{P}(z)^{*} \geq 0$ with $\check{P}(z)^{*}$ being the complex conjugate transpose of $\check{P}(z)$. Here, the state feedback controller gain is $\breve{L}(z)=\breve{R}^{-1} \breve{B}^{*} \breve{P}(z)$ for $\breve{u}(z, t)=$ $-\check{L}(z) \check{x}(z, t)$. Consequently, the matrix $\check{P}(z)$ is of the form

$$
\check{P}(z)=\left(\begin{array}{lll}
p_{11} & p_{12} & p_{13} \\
\bar{p}_{12} & p_{22} & p_{23} \\
\bar{p}_{13} & \bar{p}_{23} & p_{33}
\end{array}\right), p_{i i} \in \mathbb{R}, \quad p_{i j} \in \mathbb{C}, i \neq j,
$$

where $\bar{p}_{i j}$ is the complex conjugate of $p_{i j}$. Without loss of generality, the weighting matrices $\check{Q}$ and $\breve{R}$ are chosen to be diagonal as follows:

$$
\check{Q}(z)=\left(\begin{array}{ccc}
q_{11} & 0 & 0 \\
0 & q_{22} & 0 \\
0 & 0 & q_{33}
\end{array}\right), \quad \check{R}(z)=r,
$$

where $q_{i i} \in \mathbb{R}_{\geq 0}$ and $r \in \mathbb{R}_{>0}$. Here, $\check{Q}$ is chosen to be diagonal to penalize the states independently, which is necessary in order to take care of present singularities as will now be explained.

As can be seen in (12), for $z=1$ the derivatives of the first two states have an algebraic relation $\left(\frac{d}{d t} \check{e}(1, t)=\right.$ $\left.-h \frac{d}{d t} \breve{o}^{\prime}(1, t)\right)$. Therefore, for $z=1$, only one of the first two

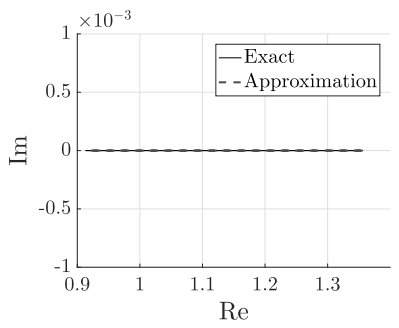

(a)

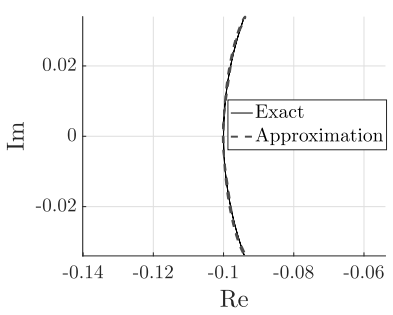

(c)

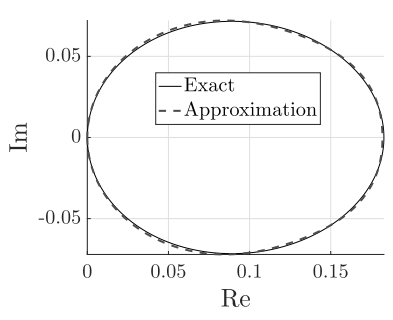

(e) (b)

(d)

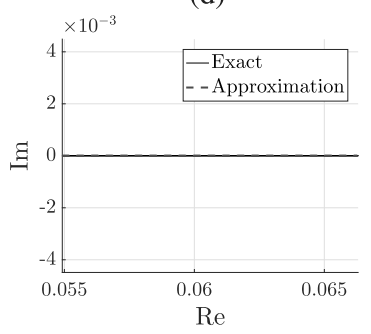

(f)
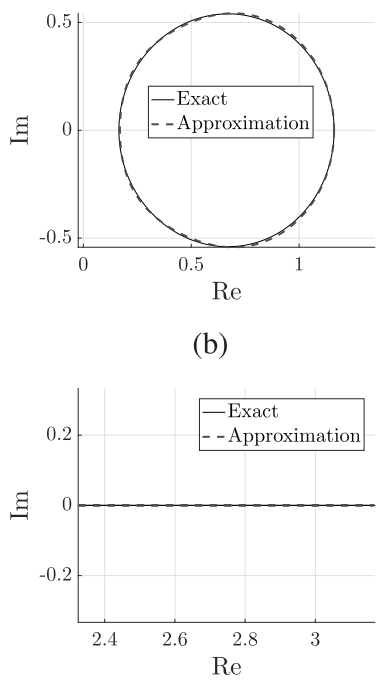

Fig. 2. Elements $p_{i j}$ of the solution to the Algebraic Riccati Equation $\check{P}(z)$ for the system (12) with the weighting matrices (17) (exact vs. least-squares approximation of degree 9) with parameter values $h=2$ and $\tau=0.1$ for $z=e^{j \theta}, \theta \in[0,2 \pi]$.

states should be weighed in the weighting matrix $\check{Q}(z)$ of the cost function (13):

$$
\check{Q}(z)=\left(\begin{array}{ccc}
1 & 0 & 0 \\
0 & \left|1-z^{-1}\right|^{2} & 0 \\
0 & 0 & 1
\end{array}\right), \quad \check{R}=1,
$$

where, the weighting matrices are chosen such that satisfying the constant time headway policy $\left(e_{k}(t) \rightarrow 0\right)$ is considered to be more important than the velocity error $\left(v_{k}^{\prime}(t) \rightarrow 0\right)$. By solving the Riccati equation (14), a solution to the optimization problem of minimizing $\breve{J}$ can be found.

The solution $\check{P}(z)$ of the Riccati equation is illustrated in Fig. 2 (exact values) for parameter values $h=2$ and $\tau=0.1$. The closed-loop poles are shown in Fig. 3 (exact values). Since there exists only one pole at the origin for $z=1$, the second condition (ii) in the Theorem VI, i.e., a countable number of poles on the imaginary axis, is satisfied and the closed-loop system is asymptotically (string) stable.

\section{B. Determining the Communication Topology By Means of Approximation}

As can be seen in Fig. 2, the analytical solution to the corresponding ARE is not easy to compute. However, the solutions in Fig. 2 seem nicely shaped, which suggests that the elements can be approximated by low-order algebraic equations in terms of $z$. To this end, each of the elements of 


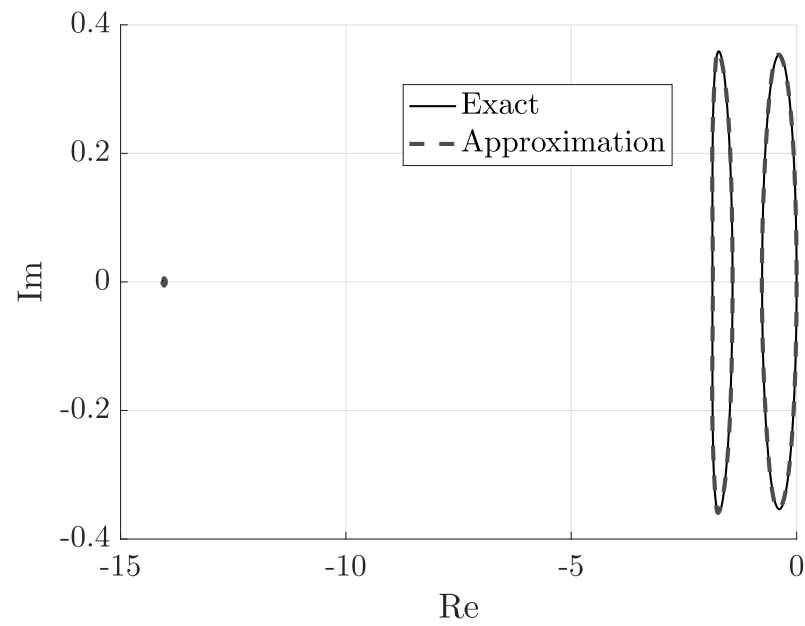

Fig. 3. Closed-loop poles for the system (12) with the weighting matrices (17) with $z=e^{j \theta}, \quad \theta \in[0,2 \pi]$. Comparison of poles obtained by least-squares approximation of degree 9 (four vehicles look-ahead/look-behind) compared to their exact values (all-to-all communication).

$\check{P}(z)$ (the $3 \times 3$ matrix function which is a solution to ARE) has to be approximated using a least-squares approach. Here, we denote the order of this approximation by $n$, which results by considering integer order of $z$ varying between $-n$ and $n$, i.e., $z^{-n}, \ldots, z^{n}$, in the least-squares method with degree $2 n+1$. The order of approximation $n$, directly affects both the state feedback and the communication topology. Namely, the state feedback operator is of the form $\check{L}(z)=\check{R}^{-1} \check{B}^{*} \check{P}(z)$ (or $\left.\breve{L}_{\text {approx }}(z)=\breve{R}^{-1} \breve{B}^{*} \breve{P}_{\text {approx }}(z)\right)$, in which $\breve{P}_{\text {approx }}(z)=$ $\sum_{i=-n}^{n} c_{i} z^{i}$. Moreover, $n$ is the order of approximation and determines the communication topology, i.e., $n$ vehicles lookahead/behind. So, as $\check{R}$ and $\breve{B}$ are $z$-independent, only the non-zero elements of $\check{P}_{\text {approx }}(z)$ determine the communication topology. For instance, the closed-loop poles and solutions to the corresponding ARE for a four vehicles look-ahead/lookbehind are demonstrated in Fig. 3 and 2, respectively. The approximated solution $\check{P}_{\text {approx }}(z)$ is plotted in Fig. 2 as well as the exact solution $\check{P}(z)$, which indicates that the approximation, involving a four vehicles look-ahead/look-behind topology, closely resembles the exact solution involving an all-to-all communication structure. Note that the least-squares approximations of $p_{i i}$ and $p_{i j}$, are chosen such that at $\theta=0$ $(z=1)$, the approximations are exact. Consequently, for the exact as well as for the approximated solution, the intersection of the eigenvalue map with the imaginary axis occurs at $z=1$. Clearly, Fig. 3 illustrates that also the closed-loop poles match well and that the truncated communication topology generates closed-loop (string) stability properties closely resembling those of the controller based on the exact solution with the all-to-all communication topology. Note that, for a poorly designed approximation, we may end up with an asymptotically unstable system, even though at $z=1$ the approximation is exact. Hence, the stability has to be assessed a posteriori, and subsequently the approximation has to be adapted if required.

\section{Lower-Order Approximation For the Communication Topology}

Above, we used a least-squares approximation of degree $9\left(z^{-4}, \ldots, z^{0}, \ldots, z^{4}\right)$, corresponding to four vehicles lookahead/ look-behind topology, which approximates the exact solution well. In practice, it may be desirable, e.g., due to bandwidth or range limitations of the (wireless) communication network, to limit the communication to even fewer neighboring vehicles. A co-design of controller and such reduced communication topology may be directly obtained by reducing the order of approximation of $\check{P}(z)$. Consequently, a tradeoff will arise from such further approximation in the sense that this will cause the closed-loop system to deviate more from its nominal optimal performance as realized by the infinite-dimensional controller.

For the communication topology of one vehicle look-ahead/ look-behind $(n=1)$ each of the elements of $\check{P}(z)$ can be approximated by a least-squares approximation of degree $3\left(z^{-1}, z^{0}, z^{1}\right)$.

As can be seen in Fig. 4, even though the closed-loop eigenvalue map corresponding to the approximated controller has been distorted slightly, all eigenvalues are still in the left half complex plane resulting in asymptotic string stability. The corresponding solution for the algebraic Riccati equation has the following form (18), as shown at the bottom of this page, where the numerical values are as follows:

$$
\begin{aligned}
& \mathcal{A}=\left[\begin{array}{ccc}
1.0961 & 0.6050 & -0.0985 \\
0.6050 & 2.7916 & 0.1025 \\
-0.0985 & 0.1025 & 0.0611
\end{array}\right] \\
& \mathcal{B}=\left[\begin{array}{ccc}
0.1298 & 0.0136 & -0.0172 \\
0.5437 & -0.2335 & -0.0862 \\
0.0157 & -0.0164 & -0.0031
\end{array}\right] .
\end{aligned}
$$

$$
\begin{aligned}
\check{P}(z) & =\left(\begin{array}{lll}
\alpha_{11}+\beta_{11} z+\gamma_{11} z^{-1} & \alpha_{12}+\beta_{12} z+\gamma_{12} z^{-1} & \alpha_{13}+\beta_{13} z+\gamma_{13} z^{-1} \\
\bar{\alpha}_{12}+\bar{\beta}_{12} \bar{z}_{1}+\bar{\gamma}_{12} \bar{z}^{-1} & \alpha_{22}+\beta_{22} z+\gamma_{22} z^{-1} & \alpha_{23}+\beta_{23} z+\gamma_{23} z^{-1} \\
\bar{\alpha}_{13}+\bar{\beta}_{13} \bar{z}+\bar{\gamma}_{13} \bar{z}^{-1} & \bar{\alpha}_{23}+\bar{\beta}_{23} \bar{z}+\bar{\gamma}_{23} \bar{z}^{-1} & \alpha_{33}+\beta_{33} z+\gamma_{33} z^{-1}
\end{array}\right) \\
& =\left[\begin{array}{lll}
\alpha_{11}+\beta_{11} z+\gamma_{11} z^{-1} & \alpha_{12}+\beta_{12} z+\gamma_{12} z^{-1} & \alpha_{13}+\beta_{13} z+\gamma_{13} z^{-1} \\
\bar{\alpha}_{12}+\bar{\gamma}_{12} z+\bar{\beta}_{12} z^{-1} & \alpha_{22}+\beta_{22} z+\gamma_{22} z^{-1} & \alpha_{23}+\beta_{23} z+\gamma_{23} z^{-1} \\
\bar{\alpha}_{13}+\bar{\gamma}_{13} z+\bar{\beta}_{13} z^{-1} & \bar{\alpha}_{23}+\bar{\gamma}_{23} z+\bar{\beta}_{23} z^{-1} & \alpha_{33}+\beta_{33} z+\gamma_{33} z^{-1}
\end{array}\right) \\
& =\underbrace{\left[\begin{array}{ccc}
\alpha_{11} & \alpha_{12} & \alpha_{13} \\
\bar{\alpha}_{12} & \alpha_{22} & \alpha_{23} \\
\bar{\alpha}_{13} & \bar{\alpha}_{23} & \alpha_{33}
\end{array}\right]}_{\mathcal{A}:=}+z \underbrace{\left[\begin{array}{ccc}
\beta_{11} & \beta_{12} & \beta_{13} \\
\bar{\gamma}_{12} & \beta_{22} & \beta_{23} \\
\bar{\gamma}_{13} & \bar{\gamma}_{23} & \beta_{33}
\end{array}\right]}_{\mathcal{B}:=}+z^{-1} \underbrace{\left[\begin{array}{ccc}
\gamma_{11} & \gamma_{12} & \gamma_{13} \\
\bar{\beta}_{12} & \gamma_{22} & \gamma_{23} \\
\bar{\beta}_{13} & \bar{\beta}_{23} & \gamma_{33}
\end{array}\right]}_{\mathcal{B}^{*}:=}=\mathcal{A}+z \mathcal{B}+z^{-1} \mathcal{B}^{*},
\end{aligned}
$$




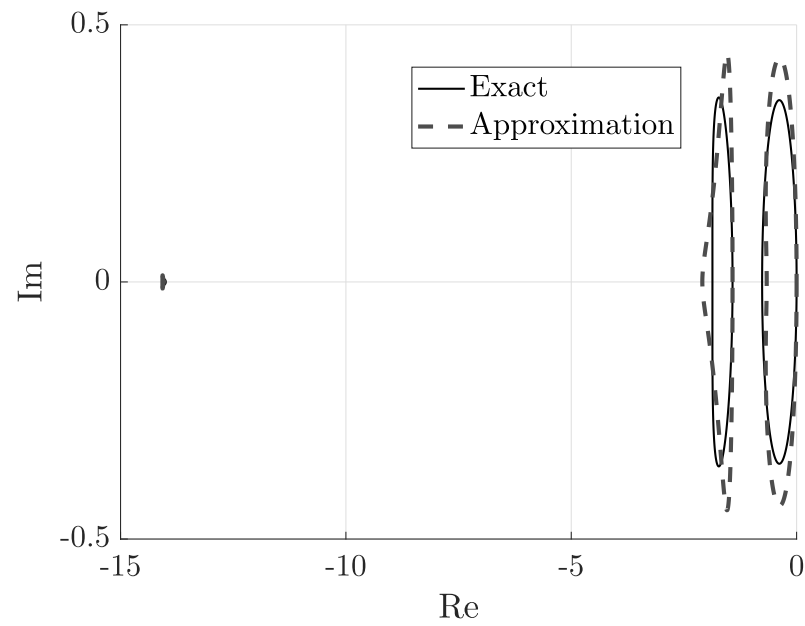

Fig. 4. Closed-loop poles for the system (12) with the weighting matrices (17) with $z=e^{j \theta}, \theta \in[0,2 \pi]$ obtained by least-squares approximation of degree 3 (one vehicle look-ahead/look-behind) compared to their exact values (all-to-all communication).

The approximated solution $\check{P}_{\text {approx }}(z)$ is plotted in Fig. 5 together with the exact solution $P(z)$, where slight deviations are noticeable. However, as mentioned before, as long as the closed-loop poles are not in the open right half plane we can ignore these differences, at least for (string) stability. Finally, the controller can be written as follows:

$$
\begin{aligned}
\check{u}(z, t) & =-\check{L}(z) \check{x}(z, t) \\
\check{L}(z) & =\check{R}^{-1} \breve{B}^{*} \check{P}(z) \\
& =\check{R}^{-1} \breve{B}^{*}\left(\mathcal{A}+z \mathcal{B}+z^{-1} \mathcal{B}^{*}\right) .
\end{aligned}
$$

\section{Implications For the Control of Finite-Length Vehicle Strings}

The state feedback $\breve{u}(z)$ designed in the $z$-domain can be interpreted in the spatial domain based on the degree of $z$. For instance, (20) can be transformed into the spatial domain via an inverse $z$-transform and show the dependency of each car to a vehicle in front and behind. Therefore, for any chosen communication topology, we can transform the obtained controller $\breve{u}(z)$ to the spatial domain and apply it on individual vehicles in a finite string of vehicles. One issue with this approach is the fact that there exist vehicles with non-existing neighbors at both ends of the finite string. So, for instance with the one vehicle look-ahead/look-behind communication topology, the first and last vehicles each miss one neighbor. One approach to overcome this difficulty is to assume the existence of a number of virtual vehicles at both ends of the string. Here, we adopt a different viewpoint. Namely, for long strings we can choose to ignore the nonexisting vehicles such that the vehicles on both ends operate only based on available information from their neighbors. This results in different closed-loop dynamics at both ends of the string, while the main part of the string is still behaving as an infinite string. We will present a related case study in the next section.

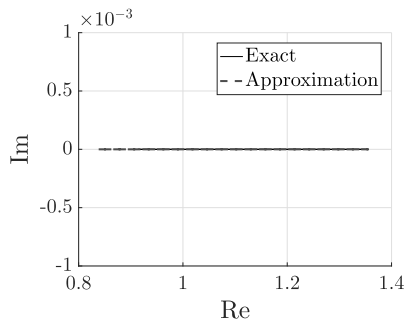

(a)

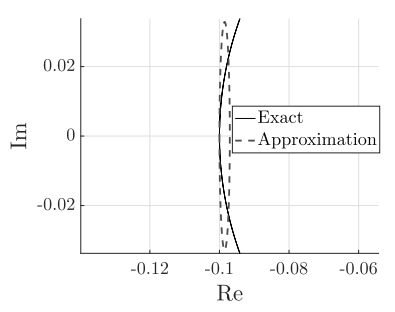

(c)

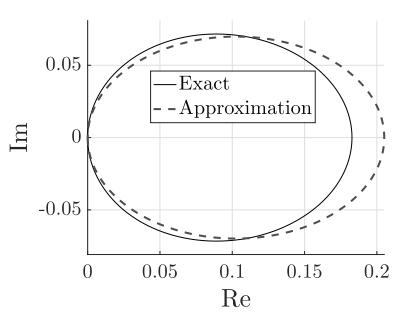

(e)

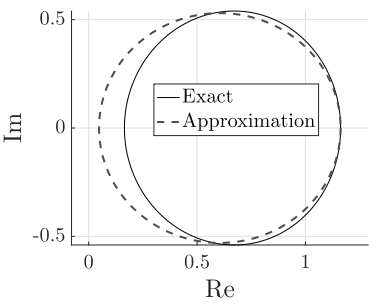

(b)

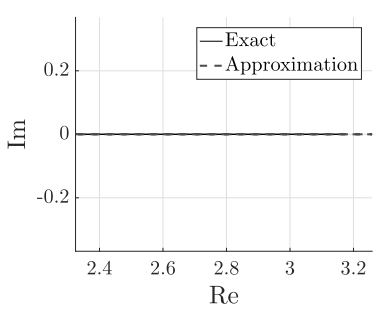

(d)

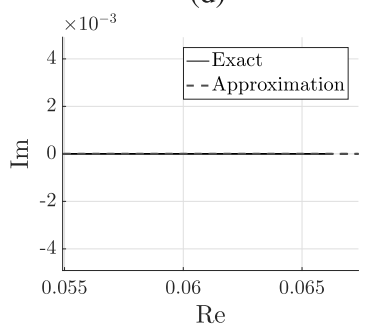

(f)
Fig. 5. Elements $p_{i j}$ of the solution to the Algebraic Riccati Equation $\check{P}(z)$ for the system (12) with the weighting matrices (17) (exact vs. least-squares approximation of degree 3) with parameter values $h=2$ and $\tau=0.1$ for $z=e^{j \theta}, \quad \theta \in[0,2 \pi]$.

\section{Simulation Results}

\section{A. Infinite-Length String Case Study}

In this set of simulations, first we consider an infinite-length string of vehicles and apply the three pairs of synthesized controllers and topologies from section IV, involving the all-to-all topology, the four vehicles look-ahead/look-behind topology, and the one vehicle look-ahead/ look-behind topology, respectively. Here, we assume that the vehicles in the string are initially in their equilibrium points except for one vehicle, which experiences an initial condition perturbation, where without loss of generality we may assume that the perturbed vehicle has index $k=0$, i.e., $x_{k}(0)=0$ for all $k \neq 0$. Then, we need to transform the initial condition vector into the $z$-domain. To this aim, a bilateral $\mathcal{Z}$-transformation (9) is applied to the initial condition vector as follows:

$$
\check{x}(z, 0)=\mathcal{Z}[x(0)](z)=\sum_{k=-\infty}^{\infty} x_{k}(0) z^{-k}=x_{0}(0) .
$$

Thereafter, we design the LQR controller with all-to-all communication using $\breve{A}(z)$ and $\breve{B}$ in (12), and $\breve{Q}(z)$ and $\check{R}$ in (17) to solve (14), which results in $\check{P}(z)$ and therefore the state feedback gain $\breve{L}(z)$. Then, by using $\check{x}(z, 0)$ in $(21)$, we simulate the string's closed-loop behavior in the $z$-domain, i.e., to obtain $\check{x}(z, t)$. Finally, we can retrieve the equivalent spatial states of any vehicle $k$ by means of the inverse 

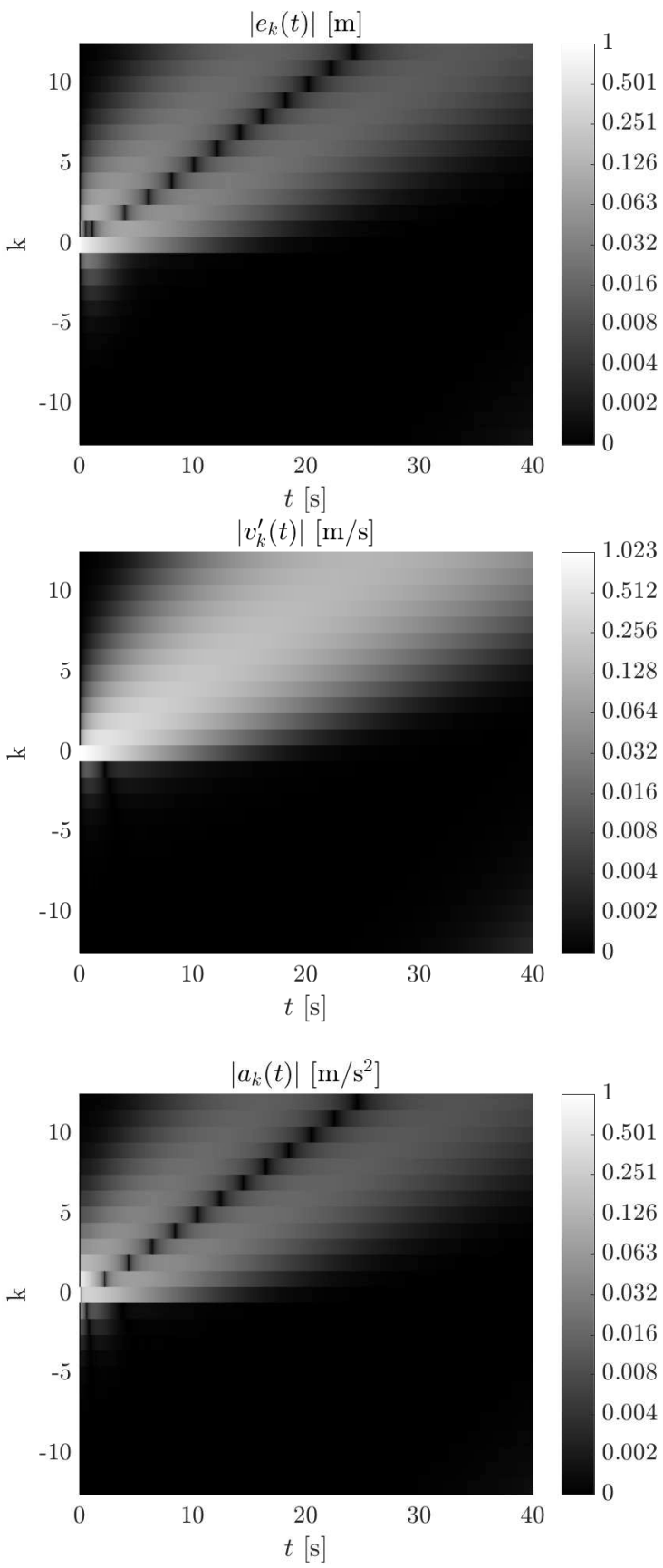

Fig. 6. Simulation results for an infinite string with all-to-all communication topology resulted from the exact solution of (14). The perturbation is introduced for vehicle $k=0$ at $t=0$. The density map is logarithmic.

bilateral $\mathcal{Z}$-transformation:

$$
\begin{aligned}
x_{k}(t) & =\frac{1}{2 \pi j} \oint \check{x}(z, t) z^{k-1} d z \\
& =\frac{1}{2 \pi j} \int_{0}^{2 \pi} \check{x}\left(e^{j \theta}, t\right) e^{j \theta(k-1)} j e^{j \theta} d \theta \\
& =\frac{1}{2 \pi} \int_{0}^{2 \pi} \check{x}\left(e^{j \theta}, t\right) e^{j \theta k} d \theta .
\end{aligned}
$$

For these simulations, we investigate the absolute value of the response in terms of the distance, velocity, and acceleration in two domains, namely, the time and the spatial index for the perturbed vehicle as well as a number of immediate neighbors in both directions. According to the definition of
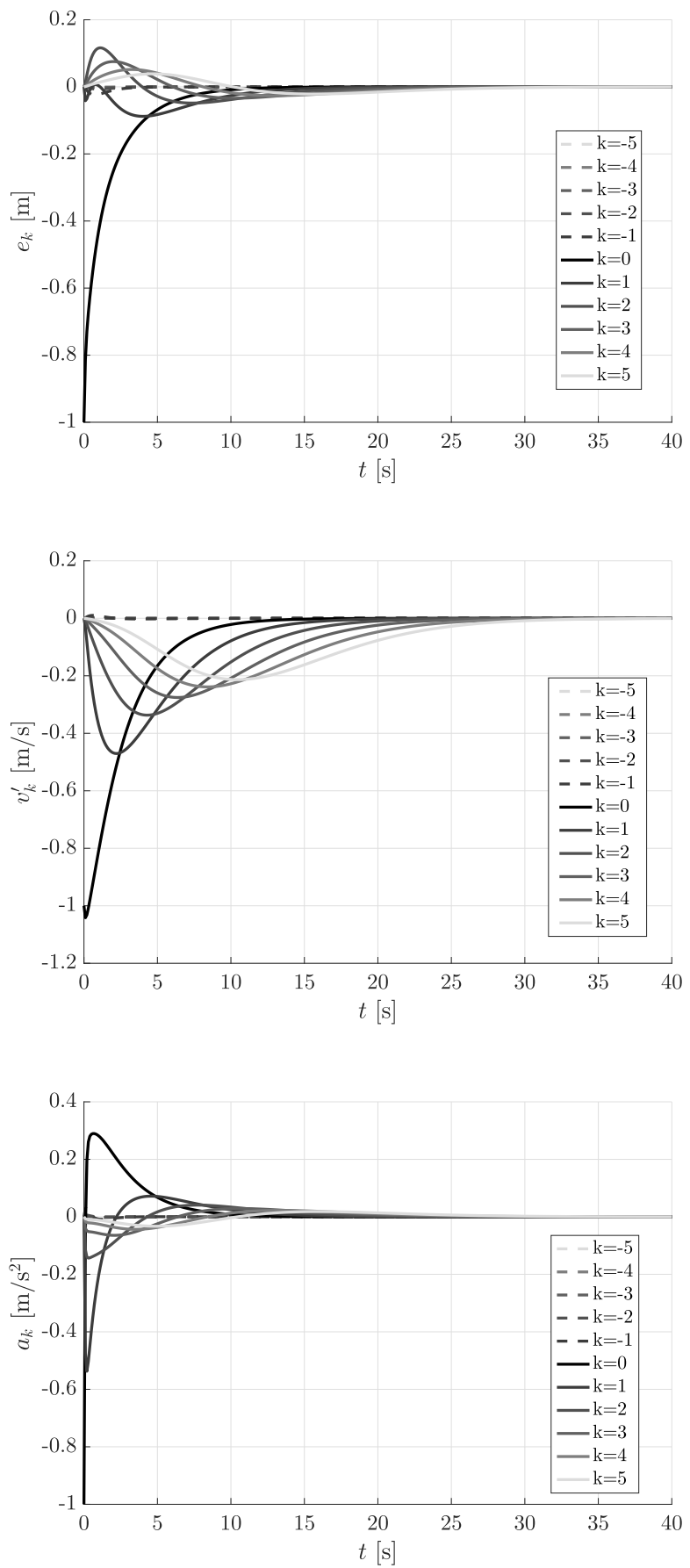

Fig. 7. Transient behavior of the vehicles with all-to-all communication topology resulted from the exact solution of (14), where the middle vehicle with index $k=0$ is disturbed at $t=0[\mathrm{~s}]$.

string stability, we expect the introduced disturbances to decay asymptotically in both dimensions. The simulation results for the case with all-to-all communication topology are illustrated in Fig. 6. Note that the density map is chosen to be logarithmic to magnify the evolution of disturbance in both directions. As can be seen, the introduced disturbances in vehicle $k=0$ at time $0[\mathrm{~s}]$, will decay along the time axis as well as the spatial index axis for all three states. Furthermore, the transient (in time) behavior of the disturbed vehicle $(k=0)$ along with ten adjacent vehicles of the string, i.e., five in front and five in behind, is captured in Fig. 7. This confirms that the states 

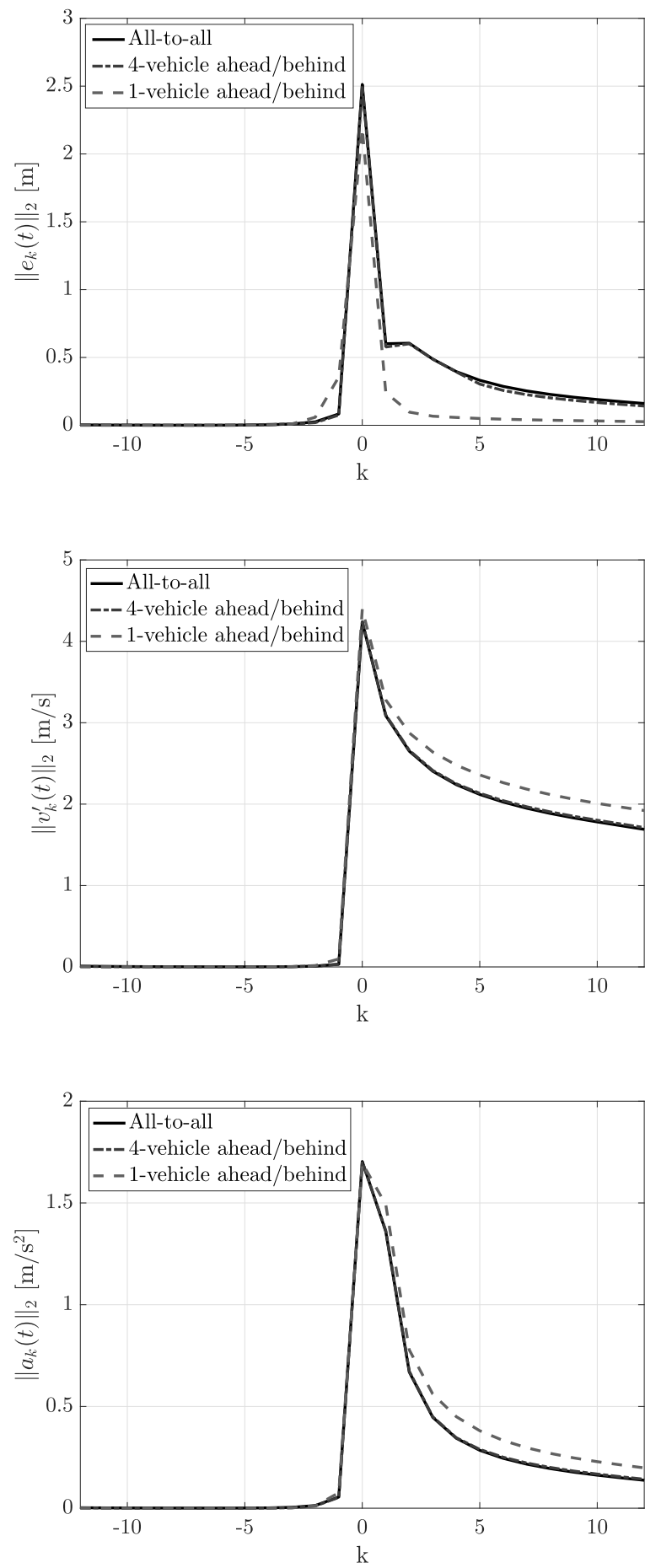

Fig. 8. Comparison between the simulation results for an infinite string of vehicles with three different communication topologies, namely, all-to-all, four vehicles look-ahead/look-behind, and one vehicle look-ahead/ look-behind.

indeed converge to zero. Similar graphs can be obtained for the other communication topologies.

To compare these three communication topologies, we may consider the signal 2-norm of the response of each vehicle with respect to time, i.e., $\left\|e_{k}(t)\right\|_{2},\left\|v_{k}^{\prime}(t)\right\|_{2},\left\|a_{k}(t)\right\|_{2}$. The results are demonstrated in Fig. 8, which confirms that all responses are decaying along the spatial dimension. As can be seen, the simulation results of the four vehicles
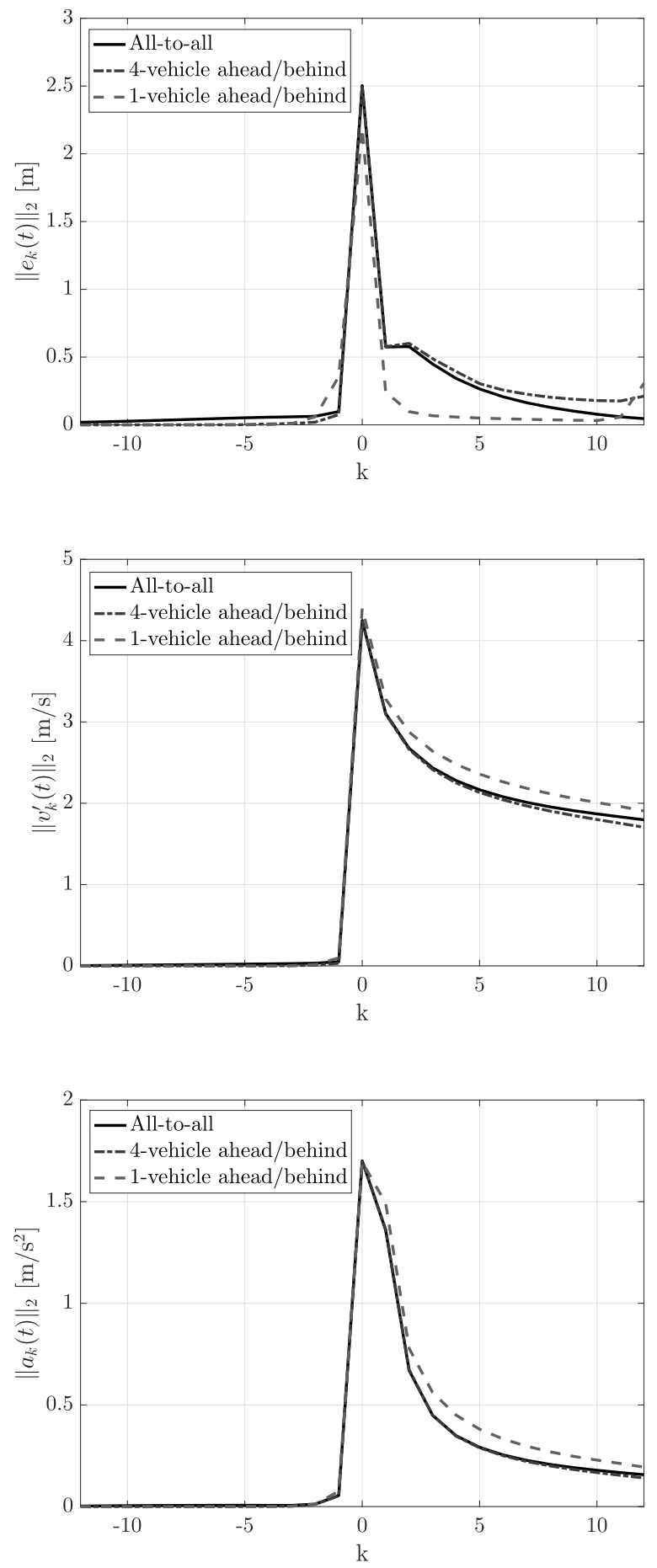

Fig. 9. Comparison between the simulation results for a finite string of vehicles with three different communication topologies, namely, all-to-all, four vehicles look-ahead/look-behind, and one vehicle look-ahead/ look-behind.

look-ahead/look-behind topology are very close to the all-to-all case compared to the one vehicle look-ahead/ look-behind case. In that sense, the four vehicles look-ahead/ look-behind strategy can be seen as a good approximation of the all-to-all strategy. Moreover, the disturbance has a small influence in the forward direction, i.e., for negative $k$, and mainly travels in the reverse (backward) direction because of the asymmetrical string dynamics in (12). In other words, 
since the dynamics of each vehicle in the string is strongly dependent on the dynamics of the vehicle directly in front (see (7)), the perturbations tend to travel in one direction. Therefore, although the communication is necessary in both directions for the string stability, the direction in which the perturbations are attenuated is dominated by the presence of the existing coupling with the directly preceding vehicle. Hence, the effects of any disturbance on any vehicle will not significantly affect the vehicles in front of it as can be seen in Fig. 6-8.

\section{B. Finite-Length Vehicle String Case Study}

We have considered a finite length string of length 25 and employed the three pairs of controllers and topologies designed for the infinite vehicle string, with the same initial conditions as used in Fig. 8. The results are demonstrated in Fig. 9. Clearly, Fig. 9 shows that the response decays away from the vehicle $k=0$ at which the (initial condition) perturbation occurs. Nevertheless, the differences corresponding topologies in Fig. 8 and Fig. 9 are noticeable at the end of vehicle string, which are due to the lack of information from non-existing vehicles. Note that, similar to the infinite string case in Fig. 6-7, the transient responses for the finite case will also converge to zero as $t \rightarrow \infty$.

\section{Discussion}

In these simulations, we employed the suggested co-design strategy in Section IV to synthesize controllers with different communication topologies for the infinite-length string with guaranteed string stability. On top of that, the results were also applied to a finite-length vehicle string with limited communication at the string ends, where no neighbors are present. For long finite strings the response will resemble the infinite-length string case, which is an indication that the suggested co-design strategy can also be useful for the finite-length vehicle strings.

\section{Vi. Conclusions}

It is known that string stability is an essential requirement for the design of vehicle following control systems that aim for short-distance following. However, commonly used approaches for analyzing string stability of a vehicular string require an already designed controller with a prioridefined communication topology. In this paper, we presented an approach for the co-design of the vehicle following controller and the communication topology jointly guaranteeing string stability, thus obtaining direct and clear insight into the effects of the communication topology on the controller performance. In this approach, the optimal control problem is considered for an infinite length vehicle string in which the vehicle string is considered as an spatially invariant system in one dimension. By solving this control problem (both in an exact an approximative sense) feedback controllers and communication topologies (varying from all-to-all to single and multiple vehicles look-ahead/ look-behind strategies) are designed simultaneously, such that asymptotic (string) stability is guaranteed. Simulation result show the effectiveness of the proposed approach. Finally, it is shown that the resulting designs also have merit in the scope of finite-length vehicle strings.

\section{APPENDIX A \\ DEFINITIONS}

Let $x(t)=\left(x_{k}(t)\right)_{k=-\infty}^{\infty}=\left(x_{-\infty}^{T}, \ldots, x_{-1}^{T}, x_{0}^{T}, x_{1}^{T}, \ldots, x_{\infty}^{T}\right)^{T}$ be a vector consisting of subvectors $x_{k}(t) \in \mathbb{C}^{n}$ with $k \in \mathbb{Z}$. The vector $x$ is said to be square summable when it lies in the $\ell_{2}$ state space, denoted by $x(t) \in \ell_{2}\left(\mathbb{C}^{n}\right)$, i.e.

Definition $2\left(\ell_{2}\right.$-state space):

$\ell_{2}\left(\mathbb{C}^{n}\right)=\left\{x(t)=\left(x_{k}(t)\right)_{k=-\infty}^{\infty} \mid x_{k}(t) \in \mathbb{C}^{n},\|x(t)\|_{\ell_{2}}<\infty\right\}$,

where $\|\cdot\|_{\ell_{2}}$ is called $\ell_{2}$-norm and it is given by

$$
\|x(t)\|_{\ell_{2}}=\sqrt{\sum_{k=-\infty}^{\infty}\left\|x_{k}(t)\right\|_{\mathbb{C}^{n}}^{2}},
$$

with $\|\psi\|_{\mathbb{C}^{n}}^{2}=\sum_{i=1}^{n}\left|\psi_{i}\right|^{2}$ for any arbitrary vector $\psi=\left(\begin{array}{c}\psi_{1} \\ \psi_{2} \\ \vdots \\ \psi_{n}\end{array}\right) \in \mathbb{C}^{n}$.

By $\mathbb{D}=\{z \in \mathbb{C}|| z \mid<1\}$, we denote the unit disk and $\partial \mathbb{D}=\{z \in \mathbb{C}|| z \mid=1\}$ denotes its boundary, the unit circle. For the given state-space $\ell_{2}\left(\mathbb{C}^{n}\right)$, the corresponding frequencydomain space equals to:

Definition $3\left(\mathcal{L}_{2}\right.$-state space):

$$
\mathcal{L}_{2}\left(\partial \mathbb{D} ; \mathbb{C}^{n}\right)=\left\{\check{x}: \partial \mathbb{D} \rightarrow \mathbb{C}^{n} \mid\|\check{x}\|_{\mathcal{L}_{2}}<\infty\right\},{ }^{4}
$$

where $\|\cdot\|_{\mathcal{L}_{2}}$ is called $\mathcal{L}_{2}$-norm and it is given by

$$
\|\check{x}\|_{\mathcal{L}_{2}}=\sqrt{\frac{1}{2 \pi} \int_{0}^{2 \pi}\left\|\check{x}\left(e^{j \theta}\right)\right\|_{\mathbb{C}^{n}}^{2} d \theta} .
$$

Note that we sometimes write $z \in \partial \mathbb{D}$, whereas other times we use its parametrization, $z=e^{j \theta}, \theta \in[0,2 \pi)$.

The $\mathcal{L}_{\infty}$-space for the operator $\check{A}(z)$ is defined as

Definition $4\left(\mathcal{L}_{\infty}\right.$-space):

$$
\begin{aligned}
\mathcal{L}_{\infty}\left(\partial \mathbb{D} ; \mathbb{C}^{n \times m}\right) & =\left\{\check{A}: \partial \mathbb{D} \rightarrow \mathbb{C}^{n \times m} \mid\right. \\
\|\check{A}\|_{\infty} & \left.=\operatorname{ess} \sup _{0<\theta \leq 2 \pi}\left\|\check{A}\left(e^{j \theta}\right)\right\|<\infty\right\},{ }^{5}
\end{aligned}
$$

where the norm $\|\check{A}\|$ is defined as $\|\check{A}\|=\sqrt{\lambda_{\max }\left(\check{A}^{*} \check{A}\right)}$, i.e. the square root of the largest eigenvalue of the positivesemidefinite matrix $\breve{A}^{*} \check{A}\left(\breve{A}^{*}\right.$ denotes the complex conjugate transpose of $\check{A}$ ) and it is called the 'largest singular value' of the matrix $\check{A}$.

\section{APPENDIX B}

\section{Stability Properties IN THE $z$-Domain}

Let us first study stability of the autonomous differential equation in the $z$-domain

$$
\frac{d}{d t} \check{x}(z, t)=\check{A}(z) \check{x}(z, t), \quad t \geq 0,
$$


where $z \in \partial \mathbb{D}$ and $\check{x}(z, t) \in \mathcal{L}_{2}\left(\partial \mathbb{D} ; \mathbb{C}^{n}\right)$ for all $t$. Exponential stability in the $z$-domain of system (28) is defined below.

Definition 5 (Exponential stability in the z-domain):

The system (28) is exponentially stable in $\mathcal{L}_{2}\left(\partial \mathbb{D} ; \mathbb{C}^{n}\right)$ if and only if there exist positive constants $M$ and $\alpha$ such that

for $\forall t \geq 0$.

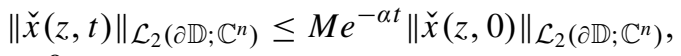

The next theorem states a necessary and sufficient condition for the exponential stability of (28) [3].

Theorem 1 (Condition for exponential stability in the z-domain): Consider the system (28) with $\check{A} \in \mathcal{L}_{\infty}\left(\partial \mathbb{D} ; \mathbb{C}^{n \times n}\right)$ and let $\breve{A}(z)$ be continuous in $z \in \partial \mathbb{D}$. Then (28) is exponentially stable if and only if for all $\theta \in[0,2 \pi]$, the matrix $\breve{A}\left(e^{j \theta}\right)$ is exponentially stable. The matrix $\breve{A}\left(e^{j \theta}\right)$ is exponentially stable if and only if

$$
\sup _{\theta \in[0,2 \pi]}\left\{\operatorname{Real} \lambda\left(\check{A}\left(e^{j \theta}\right)\right)\right\}<0
$$

where $\operatorname{det}\left(\lambda I-\check{A}\left(e^{j \theta}\right)\right)=0$ for some $\theta \in[0,2 \pi]$. In other words, the real part of all the eigenvalues of $\check{A}\left(e^{j \theta}\right)$ for all $\theta \in[0,2 \pi]$ must be in the open left-half plane.

Asymptotic stability can also be defined for the system (28) as follows.

Definition 6 (Asymptotic stability in the z-domain): The system (28) is asymptotically stable if and only if

$$
\lim _{t \rightarrow \infty}\|\check{x}(z, t)\|_{\mathcal{L}_{2}\left(\partial \mathbb{D} ; \mathbb{C}^{n}\right)}=0 \quad \forall \check{x}(z, 0) \in \mathcal{L}_{2}\left(\partial \mathbb{D} ; \mathbb{C}^{n}\right) .
$$

A condition for asymptotic stability of (28) are formalized in the next theorem [3].

Theorem 2 (Condition for asymptotic stability in the $z$-domain): The system (28) is asymptotically stable if i)

1) Real $\lambda\left(\breve{A}\left(e^{j \theta}\right)\right) \leq 0$ for all $\theta \in[0,2 \pi]$, and Real $\lambda\left(\check{A}\left(e^{j \theta}\right)\right)=0$ for at most a countable number of $\theta \in[0,2 \pi]$.

2) $\sup _{\theta \in[0,2 \pi], t \geq 0}\left\|e^{\check{A}\left(e^{j \theta}\right) t}\right\|<\infty$.

Here, the second condition $(i i)$ - which guarantees finite overshoot - is satisfied if

$$
\min _{\theta \in[0,2 \pi]}\left|\lambda_{k}\left(\check{A}\left(e^{j \theta}\right)\right)-\lambda_{l}\left(\check{A}\left(e^{j \theta}\right)\right)\right|>0 \quad k \neq l,
$$

where $k, l=1, \ldots, n$. In (32), $\lambda_{k}\left(\check{A}\left(e^{j \theta}\right)\right)$ denotes the $k$-th eigenvalue of $\breve{A}\left(e^{j \theta}\right)$ at a fixed $\theta \in[0,2 \pi]$. In other words, if (32) is satisfied, then $\breve{A}\left(e^{j \theta}\right)$ has $n$ distinct eigenvalues for any given $\theta \in[0,2 \pi]$.

\section{REFERENCES}

[1] A. A. Alam, A. Gattami, and K. H. Johansson, "An experimental study on the fuel reduction potential of heavy duty vehicle platooning," in Proc. 13th Int. IEEE Conf. Intell. Transp. Syst. (ITSC), Sep. 2010, pp. 306-311.

[2] B. Bamieh, F. Paganini, and M. A. Dahleh, "Distributed control of spatially invariant systems," IEEE Trans. Autom. Control, vol. 47, no. 7, pp. 1091-1107, Jul. 2002.

[3] R. Curtain, O. V. Iftime, and H. Zwart, "System theoretic properties of a class of spatially invariant systems," Automatica, vol. 45, no. 7, pp. 1619-1627, Jul. 2009 .
[4] R. D'Andrea and G. E. Dullerud, "Distributed control design for spatially interconnected systems," IEEE Trans. Autom. Control, vol. 48, no. 9 , pp. 1478-1495, Sep. 2003.

[5] M. Fardad and M. R. Jovanović, "Design of optimal controllers for spatially invariant systems with finite communication speed," Automatica, vol. 47, no. 5, pp. 880-889, May 2011.

[6] O. Gehring and H. Fritz, "Practical results of a longitudinal control concept for truck platooning with vehicle to vehicle communication," in Proc. IEEE Conf. Intell. Transp. Syst. (ITSC), Nov. 1997, pp. 117-122.

[7] P. Ioannou and Z. Xu, "Throttle and brake control systems for automatic vehicle following," IVHS J., vol. 1, no. 4, pp. 345-377, 1994.

[8] P. A. Ioannou and C. C. Chien, "Autonomous intelligent cruise control," IEEE Trans. Veh. Technol., vol. 42, no. 4, pp. 657-672, Nov. 1993.

[9] C.-Y. Liang and H. Peng, "Optimal adaptive cruise control with guaranteed string stability," Vehicle Syst. Dyn., vol. 31, nos. 4-5, pp. 313-330, Nov. 1999.

[10] F. Lin, M. Fardad, and M. R. Jovanović, "Optimal control of vehicular formations with nearest neighbor interactions," IEEE Trans. Autom. Control, vol. 57, no. 9, pp. 2203-2218, Sep. 2012.

[11] X. Liu, A. Goldsmith, S. S. Mahal, and J. K. Hedrick, "Effects of communication delay on string stability in vehicle platoons," in Proc. IEEE Intell. Transp. Syst., Oakland, CA, USA, Aug. 2001, pp. 625-630.

[12] G. J. L. Naus, R. P. A. Vugts, J. Ploeg, M. J. G. van de Molengraft, and M. Steinbuch, "Towards on-the-road implementation of cooperative adaptive cruise control," in Proc. 16th World Congr. Exhibit. Intell. Transp. Syst. Services, Stockholm, Sweden, Sep. 2009, pp. 1-12.

[13] G. J. L. Naus, R. P. A. Vugts, J. Ploeg, M. J. G. van de Molengraft, and M. Steinbuch, "String-stable CACC design and experimental validation: A frequency-domain approach," IEEE Trans. Veh. Technol., vol. 59, no. 9, pp. 4268-4279, Nov. 2010.

[14] J. Ploeg, B. T. M. Scheepers, E. van Nunen, N. van de Wouw, and $\mathrm{H}$. Nijmeijer, "Design and experimental evaluation of cooperative adaptive cruise control," in Proc. 14th Int. IEEE Conf. Intell. Transp. Syst. (ITSC), Washington, DC, USA, Oct. 2011, pp. 260-265.

[15] J. Ploeg, E. Semsar-Kazerooni, G. Lijster, N. van de Wouw, and H. Nijmeijer, "Graceful degradation of cooperative adaptive cruise control," IEEE Trans. Intell. Transp. Syst., vol. 16, no. 1, pp. 488-497, Feb. 2015.

[16] J. Ploeg, N. van de Wouw, and $\mathrm{H}$. Nijmeijer, " $\mathcal{L}_{p}$ string stability of cascaded systems: Application to vehicle platooning," IEEE Trans. Control Syst. Technol., vol. 22, no. 2, pp. 786-793, Mar. 2014.

[17] R. Rajamani and C. Zhu, "Semi-autonomous adaptive cruise control systems," IEEE Trans. Veh. Technol., vol. 51, no. 5, pp. 1186-1192, Sep. 2002.

[18] S. Sheikholeslam and C. A. Desoer, "Longitudinal control of a platoon of vehicles with no communication of lead vehicle information: A system level study," IEEE Trans. Veh. Technol., vol. 42, no. 4, pp. 546-554, Nov. 1993.

[19] S. S. Stankovic, M. J. Stanojevic, and D. D. Siljak, "Decentralized overlapping control of a platoon of vehicles," IEEE Trans. Control Syst. Technol., vol. 8, no. 5, pp. 816-832, Sep. 2000.

[20] D. Swaroop and J. K. Hedrick, "String stability of interconnected systems," IEEE Trans. Autom. Control, vol. 41, no. 3, pp. 349-357, Mar. 1996.

[21] D. Swaroop, J. K. Hedrick, and S. B. Choi, "Direct adaptive longitudinal control of vehicle platoons," IEEE Trans. Veh. Technol., vol. 50, no. 1 pp. 150-161, Jan. 2001.

[22] A. Vahidi and A. Eskandarian, "Research advances in intelligent collision avoidance and adaptive cruise control," IEEE Trans. Intell. Transp. Syst., vol. 4, no. 3, pp. 143-153, Sep. 2003.

[23] B. van Arem, C. J. G. van Driel, and R. Visser, "The impact of cooperative adaptive cruise control on traffic-flow characteristics," IEEE Trans. Intell. Transp. Syst., vol. 7, no. 4, pp. 429-436, Dec. 2006.

[24] P. Venhovens, K. Naab, and B. Adiprasito, "Stop and go cruise control," Int. J. Autom. Control, vol. 1, no. 2, pp. 61-69, 2000.

[25] H. Zwart, A. Firooznia, J. Ploeg, and N. van de Wouw, "Optimal control for non-exponentially stabilizable spatially invariant systems with an application to vehicular platooning," in Proc. IEEE 52nd Annu. Conf. Decision Control (CDC), Dec. 2013, pp. 3038-3042. 


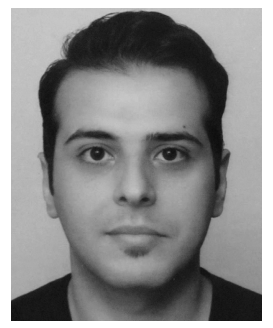

Amir Firooznia (S'13) was born in 1988 $\mathrm{He}$ received the B.Sc. degree in aerospace engineering from Sharif University of Technology, Tehran, Iran, in 2010 and the M.Sc. degree in mechanical engineering from Eindhoven University of Technology, Eindhoven, The Netherlands, in 2012.

$\mathrm{He}$ is with the Delft Center for Systems and Control Department, Delft University of Technology, Delft, The Netherlands. His interests include distributed control and optimization.

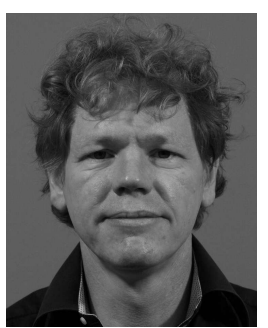

Jeroen Ploeg received the M.Sc. degree in mechanical engineering from Delft University of Technology, Delft, The Netherlands, in 1988 and the Ph.D. degree in mechanical engineering on the control of vehicle platoons from Eindhoven University of Technology, Eindhoven, The Netherlands, in 2014.

From 1989 to 1999 , he was with Koninklijke Hoogovens (currently Tata Steel), IJmuiden, The Netherlands, where his interest was the development and implementation of dynamic process control systems for large-scale industrial plants. Since 1999, he has been a Senior Research Scientist with the Integrated Vehicle Safety Department, TNO, Helmond, The Netherlands. His interests include control system design for cooperative and automated vehicles in general and automated vehicle platoons in particular, the design of interaction protocols for complex driving scenarios, and motion control of wheeled mobile robots. This research is executed in close cooperation with the Department of Mechanical Engineering, Eindhoven University of Technology. He is an Associate Editor of IEEE TRANSACTIONS ON INTELLIGENT TRANSPORTATION SYSTEMS.

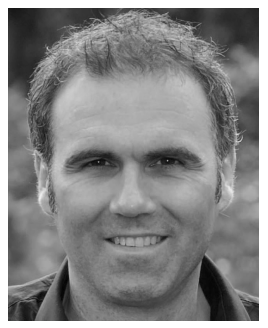

Nathan van de Wouw (M’08) was born in 1970. $\mathrm{He}$ received the M.Sc. degree (with Hons.) and the Ph.D. degree in mechanical engineering from Eindhoven University of Technology, Eindhoven, The Netherlands, in 1994 and 1999, respectively.

He currently holds a full professor position with the Mechanical Engineering Department, Eindhoven University of Technology. He also holds an adjunct full professor position with University of Minnesota, USA, and a (part-time) full professor position with Delft University of Technology, The Netherlands. In 2000, he was with Philips Applied Technologies, Eindhoven. In 2001, he was with TNO, the Netherlands Organization for Applied Scientific Research, Delft, The Netherlands. He has held positions as a visiting professor with University of California at Santa Barbara, Santa Barbara, USA, in 2006/2007, with University of Melbourne, Australia, in 2009/2010, and with University of Minnesota, USA, in 2012 and 2013. He has authored a large number of journal and conference papers and the books Uniform Output Regulation of Nonlinear Systems: A Convergent Dynamics Approach (Birkhauser, 2005), with A.V. Pavlov and H. Nijmeijer, and Stability and Convergence of Mechanical Systems with Unilateral Constraints (Springer-Verlag, 2008) with R.I. Leine. His research interests are the analysis and control of nonlinear/hybrid systems, with applications to vehicular platooning, high-tech systems, resource exploration, smart energy systems, and networked control systems.

Dr. van de Wouw received the IEEE Control Systems Technology Award For the development and application of variable-gain control techniques for high performance motion systems. He is an Associate Editor of the journals Automatica and IEEE TRANSACTIONS ON CONTROL Systems TECHNOLOGY.

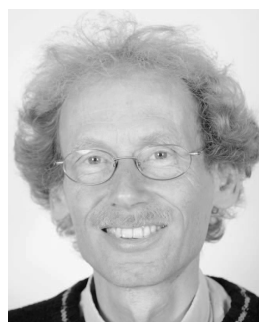

Hans Zwart (M'11) was born in HoogezandSappemeer, The Netherlands, in 1959. He received the Drs. degree in mathematics from University of Groningen, in 1984 and the Ph.D. degree in 1988.

Since 1988, he has been with the Department of Applied Mathematics, University of Twente, Enschede, The Netherlands, currently as a Full Professor. Since 2011, he also has a one-day-perweek appointment within Mechanical Engineering Department, Eindhoven University of Technology. Together with R. Curtain, he has authored a standard reference in the field of infinite-dimensional systems theory. His research interest is the control of distributed parameter system, with presently an emphasis on port-Hamiltonian systems and infinite platoons. 